CHRONIC OBSTRUCTIVE PULMONARY DISEASE

\title{
C-reactive protein and mortality in mild to moderate chronic obstructive pulmonary disease
}

\author{
S F P Man, J E Connett, N R Anthonisen, R A Wise, D P Tashkin, D D Sin
}

See end of article for authors' affiliations

.....................

Correspondence to: Dr D D Sin, James Hogg iCAPTURE Center for Cardiovascular and Pulmonary Research, St Paul's Hospital, 1081 Burrard Street, Vancouver BC, Canada V6Z 1Y6; dsin@mrl.ubc.ca

Received 26 January 2006 Accepted 16 May 2006

Published Online First

31 May 2006
Background: Although C-reactive protein (CRP) levels are increased in chronic obstructive pulmonary disease (COPD), it is not certain whether they are associated with adverse clinical outcomes.

Methods: Serum CRP levels were measured in 4803 participants in the Lung Health Study with mild to moderate COPD. The risk of all-cause and disease specific causes of mortality was determined as well as cardiovascular event rates, adjusting for important covariates such as age, sex, cigarette smoking, and lung function. Cardiovascular events were defined as death from coronary heart disease or stroke, or nonfatal myocardial infarction or stroke requiring admission to hospital.

Results: CRP levels were associated with all-cause, cardiovascular, and cancer specific causes of mortality. Individuals in the highest quintile of CRP had a relative risk (RR) for all-cause mortality of $1.79195 \%$ confidence interval (CI) 1.25 to 2.56) compared with those in the lowest quintile of CRP. For cardiovascular events and cancer deaths the corresponding RRs were 1.51 (95\% $\mathrm{Cl} 1.20$ to 1.90$)$ and $1.85(95 \% \mathrm{Cl} 1.10$ to 3.13), respectively. CRP levels were also associated with an accelerated decline in forced expiratory volume in 1 second $(p<0.001)$. The discriminative property of CRP was greatest during the first year of measurement and decayed over time. Comparing the highest and lowest CRP quintiles, the RR was $4.03(95 \% \mathrm{Cl} 1.23$ to 13.21 ) for 1 year mortality, 3.30 (95\% Cl 1.38 to 7.86 ) for 2 year mortality, and $1.82(95 \% \mathrm{Cl} 1.22$ to 2.68$)$ for $\geqslant 5$ year mortality.

Conclusions: CRP measurements provide incremental prognostic information beyond that achieved by traditional markers of prognosis in patients with mild to moderate COPD, and may enable more accurate detection of patients at a high risk of mortality.

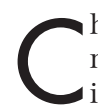
hronic obstructive pulmonary disease (COPD) is a major global epidemic, affecting $5-15 \%$ of all adults in industrialised countries ${ }^{1}$ and accounting for over 3 million deaths each year worldwide. ${ }^{2}$ COPD is an inflammatory disorder characterised by progressive airflow limitation and parenchymal destruction. ${ }^{1}$ The inflammatory process may extend beyond the pulmonary system, resulting in a state of persistent low grade systemic inflammation ${ }^{3}$ which has been implicated in various complications of COPD including cachexia, ${ }^{5}$ ischaemic heart disease, ${ }^{6}$ and arrhythmias. $^{78}$ A recent study indicated that plasma fibrinogen levels are associated with an accelerated decline in lung function; ${ }^{9}$ however, to date, there is a scarcity of longitudinal data that have temporally linked systemic inflammation with hard clinical end points such as mortality and hospital admissions in patients with COPD. Using the Lung Health Study (LHS) cohort, $^{10}$ we determined whether serum Creactive protein (CRP), a marker of systemic inflammation, is associated with (1) increased risk of all-cause and disease specific causes of mortality, (2) increased risk of fatal and non-fatal cardiovascular events, and (3) an accelerated decline in lung function as measured by forced expiratory volume in 1 second $\left(\mathrm{FEV}_{1}\right)$. The relationship between CRP and cardiovascular events is very germane because they are the leading causes of hospital admissions and mortality in patients with mild to moderate COPD. ${ }^{11}$

\section{METHODS}

\section{Study participants}

The study design, spirometric techniques, smoking intervention programmes, and methods used for collection of vital status and hospitalisation data in the original Lung Health Study (LHS) have been reported previously. ${ }^{11}$ Briefly, the LHS recruited 5887 cigarette smoking participants aged
35-60 years with mild to moderate airflow obstruction on spirometric testing. Individuals who had a history of cancer (except carcinoma in situ or basal cell carcinoma of the skin), myocardial infarction (in the past 2 years), angina, heart failure, stroke (in the past 2 years), renal failure, cirrhosis or other serious liver diseases, pulmonary embolism, disorders of the central nervous system, narrow angle glaucoma, or any other major diseases which could have compromised follow up were excluded from the cohort.

Mild to moderate airflow obstruction was defined as $\mathrm{FEV}_{1}$ $<90 \%$ predicted but $\geqslant 55 \%$ predicted in the presence of $\mathrm{FEV}_{\mathrm{l}} /$ forced vital capacity (FVC) ratio of $<0.70$. After enrolment and baseline measurements, the study participants were asked to visit the study centre annually for follow up. At these visits spirometric parameters were measured before and after two puffs of isoproteronol. The largest single FEV 1 and FVC were reported and converted to percentages of the predicted normal value using the formula of Crapo and coworkers. ${ }^{12}$ In addition, the health status, symptoms, and health service usage of participants over the preceding 12 months were captured using a detailed questionnaire. At these visits salivary cotinine levels were measured to verify objectively the smoking status of the participants. Participants were categorised as sustained quitters if they were biochemically validated non-smokers at each annual visit. Participants who were smokers at each annual visit were continuing smokers. Those whose behaviour varied were classified as intermittent quitters. The initial LHS involved 5 years of follow up and the results of this phase of the study have been published previously. ${ }^{10}{ }^{11}$

Abbreviations: $\mathrm{BMI}$, body mass index; $\mathrm{COPD}$, chronic obstructive pulmonary disease; CRP, C-reactive protein; $\mathrm{FEV}_{1}$, forced expiratory volume in 1 second; FVC, forced vital capacity 
At the fifth annual visit a venipuncture was carried out on participants who attended their LHS clinics. At year 5, 5413 participants were alive and were eligible for venipuncture. Of these, 4803 provided serum samples (89\% of eligible participants). At this visit the participants were also asked to consent for additional follow up (LHS 3). During the follow up of LHS 3, the vital status and hospitalisation records of participants were captured biannually. If a participant had been admitted to hospital, copies of essential documents were obtained from hospital record rooms. Records that made significant mention of respiratory disease, cardiovascular disease, or cancer were forwarded to the mortality and morbidity review board of the study for definitive coding. The mortality and morbidity review board was also responsible for classifying the causes of death for all participants who died during the study. They reviewed death certificates, necropsy reports, relevant hospital records, and summaries of interviews with attending physicians or eye witnesses. These data were supplemented by linkages with a National Death Index which provided the date and cause of death for all US study participants through the end of 2001. Vital status was successfully determined for $98.3 \%$ of the participants. ${ }^{13}$ Mortality end points were classified into: coronary heart disease, cardiovascular disease (which also included coronary heart disease), lung cancer, other cancer, respiratory disease excluding lung cancer, other, and unknown.

\section{Measurements of CRP}

After collection the blood samples were separated into their various components. They were then shipped to the LHS data coordinating centre on dry ice and kept in $-70^{\circ} \mathrm{C}$ freezers until use. The serum samples were thawed once for CRP determination. The levels of CRP were measured using highly sensitive enzyme linked immunosorbent assay kits (Alpha Diagnostics, San Antonio, TX, USA). All assays were measured at the James Hogg iCAPTURE Center (University of British Columbia, Vancouver, Canada) by laboratory personnel who were unaware of the clinical outcomes of the study participants. All assays were performed in triplicate. The median interassay coefficient of variation was $6.3 \%$.

\section{Statistical analysis}

The primary relationship of interest was between serum CRP levels and all-cause mortality. Other end points were considered secondary in nature. All individuals who died or were lost to follow up before visit 5 were censored. CRP values were divided into quintiles from the lowest to the highest levels. We compared the risk of all-cause mortality across the CRP quintiles over the follow up period using a Cox proportional hazards model in which the following covariates were adjusted for: sex, race, age (in quintiles), body mass index (BMI) (in quintiles), pack-years of smoking (in quintiles), biochemically validated smoking status (continued smokers, sustained quitters, or intermittent quitters), rate of decline in $\mathrm{FEV}_{1}$ during the first 5 years of follow up (in quintiles), and percentage predicted $\mathrm{FEV}_{1}$ (in quintiles). To determine the relationship between CRP and cardiovascular disease events, defined as either fatal or non-fatal cardiovascular disease hospital admissions, we used the same model as for all-cause mortality. Multiple regression modelling was used to determine the relationship between CRP quintiles and the rate of decline in $\mathrm{FEV}_{1}$. We have previously shown in this cohort that the slope of $\mathrm{FEV}_{1}$ decline over the first 5 years of follow up reliably predicts the rate of decline over much longer periods of follow up (over 11 years). ${ }^{14}$ Because CRP was non-normally distributed, for certain analyses we log transformed CRP values to achieve normality.

To determine whether the predictive (discriminative) power of baseline CRP levels decayed over time, we ran a series of logistic regression models in which we determined all-cause mortality rates from 1 to $>5$ years of follow up. From these models we determined the area under the receiver operating characteristic curve (also known as C statistic) of baseline CRP. The $\mathrm{C}$ statistic can range from 0.5 (model discrimination no better than by chance) to 1.0 (perfect model discrimination). We then constructed a multiple logistic regression model in which sex, race, age (in quintiles), BMI (in quintiles), biochemically validated smoking status, and percentage predicted $\mathrm{FEV}_{1}$ (in quintiles), together with serum CRP levels, were included as potential covariates to predict 1 year mortality. We used a stepwise selection process to select variables that had a $p$ value of 0.20 or less in the univariate model and 0.10 or less in the multivariate model. ${ }^{15}$ Discrimination of the model was assessed by the $\mathrm{C}$ statistic and calibration was assessed using the Hosmer and Lemeshow $\chi^{2}$ statistic $(p>0.05$ for all models). We also did this for longer term mortality but the discriminative power of baseline measurements diminished significantly over time.

Continuous variables are presented as mean (SD) unless otherwise specified. All analyses were performed using SAS software version 9.1 (SAS Institute, Carey, NC, USA). p

Table 1 Characteristics of participants in the Lung Health Study according to quintiles of baseline CRP levels

\begin{tabular}{|c|c|c|c|c|c|c|}
\hline & $\begin{array}{l}\text { Quintile } 1 \\
(n=960)\end{array}$ & $\begin{array}{l}\text { Quintile } 2 \\
\text { (n=961) }\end{array}$ & $\begin{array}{l}\text { Quintile } 3 \\
\text { (n=961) }\end{array}$ & $\begin{array}{l}\text { Quintile } 4 \\
(n=961)\end{array}$ & $\begin{array}{l}\text { Quintile } 5 \\
(\mathrm{n}=960)\end{array}$ & p for trend* \\
\hline CRP $(\mathrm{mg} / \mathrm{l}) \dagger$ & $0.21(0.17-0.39)$ & $0.73(0.62-0.86)$ & $1.39(1.21-1.61)$ & $2.58(2.17-3.06)$ & $7.06(4.79-9.02)$ & $<0.001$ \\
\hline Age (years) & $52(7)$ & $53(7)$ & $54(7)$ & $54(7)$ & $55(6)$ & $<0.001$ \\
\hline Men & $568(59 \%)$ & $617(64 \%)$ & $660(69 \%)$ & $630(66 \%)$ & $548(57 \%)$ & 0.568 \\
\hline Whites & $932(97 \%)$ & $936(97 \%)$ & $933(97 \%)$ & $925(96 \%)$ & $897(93 \%)$ & $<0.001$ \\
\hline Pack-years of smoking & $37(18)$ & $39(18)$ & $41(18)$ & $42(20)$ & $42(19)$ & $<0.001$ \\
\hline Continued smokers & $470(49 \%)$ & $509(53 \%)$ & $509(53 \%)$ & $536(56 \%)$ & $577(60 \%)$ & $<0.001$ \\
\hline Intermittent smokers & $296(31 \%)$ & $275(28 \%)$ & $269(28 \%)$ & $267(28 \%)$ & $244(25 \%)$ & 0.011 \\
\hline Daily cough & $317(33 \%)$ & $341(35 \%)$ & $344(36 \%)$ & $333(35 \%)$ & $352(37 \%)$ & 0.185 \\
\hline Daily sputum & $273(28 \%)$ & $287(30 \%)$ & $306(32 \%)$ & $287(30 \%)$ & $313(33 \%)$ & 0.076 \\
\hline $\mathrm{BMI}\left(\mathrm{kg} / \mathrm{m}^{2}\right)$ & $24.1(3.6)$ & $25.2(3.6)$ & $25.6(3.5)$ & $26.3(4.0)$ & $26.6(4.2)$ & $<0.001$ \\
\hline $\mathrm{FEV}_{1}(\mathrm{I}) \ddagger$ & $2.80(0.63)$ & $2.80(0.64)$ & $2.80(0.63)$ & $2.74(0.63)$ & $2.61(0.59)$ & $<0.001$ \\
\hline $\mathrm{FEV}_{1}(\%$ predicted) $)$ & $80(9)$ & $79(9)$ & $78(9)$ & $78(10)$ & $77(9)$ & $<0.001$ \\
\hline Diastolic BP & $76(9)$ & $78(9)$ & $79(9)$ & 79 (9) & $79(9)$ & $<0.001$ \\
\hline Systolic BP & $119(14)$ & $121(15)$ & $123(15)$ & $123(14)$ & 125 (16) & $<0.001$ \\
\hline
\end{tabular}

$\mathrm{CRP}, \mathrm{C}$-reactive protein; $\mathrm{BMI}$, body mass index; $\mathrm{FEV}_{1}$, forced expiratory volume in 1 second; $\mathrm{BP}$, blood pressure.

*Linear trend from quintile 1 to quintile 5 .

†Geometric mean (interquartile range).

†Post-bronchodilator values.

Continuous variables are presented as mean (SD) and dichotomous variables are presented as number of participants (\% column totals) unless otherwise indicated. 
Table 2 Clinical outcomes of participants in the Lung Health Study according to quintiles of baseline CRP levels

\begin{tabular}{|c|c|c|c|c|c|c|}
\hline & $\begin{array}{l}\text { Quintile } 1 \\
(n=960)\end{array}$ & $\begin{array}{l}\text { Quintile } 2 \\
\text { ( } n=961)\end{array}$ & $\begin{array}{l}\text { Quintile } 3 \\
\text { (n=961) }\end{array}$ & $\begin{array}{l}\text { Quintile } 4 \\
\text { (n=961) }\end{array}$ & $\begin{array}{l}\text { Quintile } 5 \\
(n=960)\end{array}$ & $p$ for trend $t$ \\
\hline Mean CRP $(\mathrm{mg} / \mathrm{l})^{*}$ & 0.21 & 0.73 & 1.39 & 2.58 & 7.06 & \\
\hline Total deaths & $47(4.9)$ & $50(5.2)$ & $61(6.4)$ & $65(6.8)$ & $106(11.0)$ & $<0.001$ \\
\hline CHD deaths & $7(0.7)$ & $5(0.5)$ & $8(0.8)$ & $9(0.9)$ & $22(2.3)$ & 0.001 \\
\hline CVD deaths & $13(1.4)$ & $7(0.7)$ & $17(1.8)$ & $17(1.8)$ & $33(3.4)$ & $<0.001$ \\
\hline Fatal/non-fatal CHD & $68(7.1)$ & $85(8.8)$ & $86(9.0)$ & $119(12.4)$ & $145(15.1)$ & $<0.001$ \\
\hline Fatal/non-fatal CVD & $120(12.5)$ & $133(13.8)$ & $147(15.3)$ & $187(19.5)$ & $237(24.7)$ & $<0.001$ \\
\hline Cancer deaths & $21(2.2)$ & $32(3.3)$ & $29(3.0)$ & $37(3.9)$ & $50(5.2)$ & 0.001 \\
\hline Lung cancer deaths & $13(1.4)$ & $23(2.4)$ & $15(1.6)$ & $20(2.1)$ & $28(2.9)$ & 0.053 \\
\hline Respiratory deaths & $8(0.8)$ & $3(0.3)$ & $6(0.6)$ & $3(0.3)$ & $6(0.6)$ & 0.578 \\
\hline
\end{tabular}

$\mathrm{CHD}$, coronary heart disease; CVD, cardiovascular disease.

${ }^{*}$ Geometric mean value for each quintile of CRP.

†Linear trend from quintile 1 to quintile 5.

Variables presented as number of participants (\% column totals) unless otherwise indicated.

values were two tailed and those below 0.05 were considered to indicate statistical significance.

\section{RESULTS}

The mean (SD) age of the participants was 53 (7) years. Their $\mathrm{FEV}_{1}$ was 2.75 (0.63) l (78 (9)\% predicted), BMI was 25.6 (3.9) $\mathrm{kg} / \mathrm{m}^{2}$, and the mean smoking history was 40 (19) pack-years. Of the participants, 3023 (63\%) were men, 4623 (96\%) were white, 2601 (54\%) were continued smokers, 1351 $(28 \%)$ were intermittent quitters, and the rest were sustained quitters on the basis of salivary cotinine measurements at visit 5. The geometric mean serum CRP level was $1.32 \mathrm{mg} / \mathrm{l}$ (interquartile range 0.62-3.06). During the follow up period, $329(6.8 \%)$ participants died; $87(26 \%)$ from cardiovascular disease, 99 (31\%) from lung cancer, 26 (8\%) from respiratory failure, and the rest from other causes $(\mathrm{n}=117 ; 36 \%)$. During the follow up period, 821 participants were admitted to hospital for cardiovascular diseases (17\%). The median duration of follow up was 7.5 years from the date of venipuncture (visit 5).

The clinical characteristics of the study participants divided into quintiles of CRP are summarised in table 1. Participants in the higher CRP quintiles were slightly older and heavier. Moreover, participants who had higher CRP levels were more likely to be continued or intermittent smokers than those with lower CRP levels. The prevalence of cough or sputum production was similar between the groups. The risk of mortality over the follow up period increased as a function of CRP quintile. Both cancer and cardiovascular causes of mortality increased along the CRP gradient (table 2).

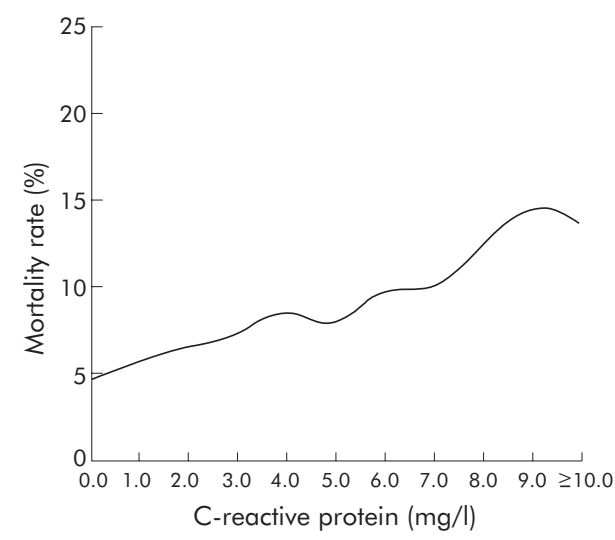

Figure 1 Risk for all-cause mortality as a function of baseline $\mathrm{C}$-reactive protein levels in the Lung Health Study cohort. The curve was fitted using a cubic spline technique.
Similarly, the risk of fatal and non-fatal coronary heart disease and cardiovascular diseases also increased along the CRP quintile gradient. However, the risk of respiratory deaths was similar between the groups.

After adjustment for potential confounders (see Methods section for details), the risk of all-cause mortality increased significantly as a function of serum CRP levels (table 3 ). Similarly, the risk of fatal and non-fatal coronary heart disease and cardiovascular diseases and cancer specific mortality increased along the CRP gradient. Respiratory causes of mortality, however, were not significantly related to serum CRP levels. To determine whether the relationship between CRP and all-cause mortality was threshold-dependent or continuous, we plotted a cubic spline curve. Figure 1 shows that the relationship between CRP and all-cause mortality is linear over the range of CRP that is prevalent in patients with mild to moderate COPD (0-6 mg/l).

Table 4 summarises the rate of decline in $\mathrm{FEV}_{1}$ (percentage predicted) as a function of serum CRP levels. The highest quintile had the fastest decline, while the lowest quintile had the slowest decline in all smoking categories. This relationship was not materially modified by smoking status $(\mathrm{p}=0.140)$.

For 1 year mortality, the $\mathrm{C}$ statistic for CRP (in quintiles) was 0.69 (95\% CI 0.58 to 0.81 ). Quintiles of age had a C statistic of 0.70 (95\% CI 0.57 to 0.82 ), while $\mathrm{FEV}_{1}$ (in quintiles) had a C statistic of 0.65 (95\% CI 0.53 to 0.77 ). In a multiple regression model (described in the Methods section), age $(p=0.002)$, race $(p<0.001)$, BMI $(p=0.005)$, and CRP levels $(p=0.003)$ were the only variables that significantly predicted 1 year mortality. When combined, these variables had a $\mathrm{C}$ statistic of 0.82 indicating excellent discriminative power. The discriminative power of CRP decreased over time. The $\mathrm{C}$ statistic of CRP alone was 0.64 ( $95 \%$ CI 0.56 to 0.72 ) for 2 year mortality, 0.63 (0.59 to 0.69 ) for 3 year mortality, 0.59 (95\% CI 0.54 to 0.65 ) for 4 year mortality, 0.60 ( $95 \%$ CI 0.55 to 0.64 ) for 5 year mortality, and 0.59 (95\% CI 0.55 to 0.62 ) for $>5$ year mortality. Comparing the highest with the lowest CRP quintile, the RR was 4.03 (95\% CI 1.23 to 13.21 ) for l year mortality, 3.30 (95\% CI 1.38 to 7.86 ) for 2 year mortality, 2.77 (95\% CI 1.46 to 5.27 ) for 3 year mortality, 1.98 (95\% CI 1.22 to 3.24 ) for 4 year mortality, and 1.82 (95\% CI 1.22 to 2.68 ) for $\geqslant 5$ year mortality.

\section{DISCUSSION}

In this large prospective study of over 4800 individuals with mild to moderate COPD, serum CRP levels were found to be a significant predictor of all-cause mortality. The risk increased linearly along the CRP gradient, even after adjustments for 
Table 3 Adjusted risk of clinical outcomes in the Lung Health Study cohort according to quintiles of baseline CRP levels

\begin{tabular}{|c|c|c|c|c|c|c|}
\hline & Quintile 1 & Quintile 2 & Quintile 3 & Quintile 4 & Quintile 5 & $\begin{array}{l}p \text { for } \\
\text { trendt }\end{array}$ \\
\hline Mean CRP $(\mathrm{mg} / \mathrm{l})^{*}$ & 0.21 & 0.73 & 1.39 & 2.58 & 7.06 & \\
\hline All-cause mortality & 1.0 & $0.98(0.65$ to 1.46$)$ & $1.14(0.78$ to 1.68$)$ & $1.13(0.77$ to 1.65$)$ & 1.79 (1.25 to 2.56$)$ & $<0.001$ \\
\hline CHD deaths & 1.0 & 0.56 (0.18 to 1.79$)$ & $0.93(0.34$ to 2.60$)$ & $0.87(0.32$ to 2.39$)$ & 2.20 (0.90 to 5.38$)$ & 0.016 \\
\hline CVD deaths & 1.0 & 0.43 (0.17 to 1.09$)$ & $1.08(0.52$ to 2.34$)$ & $0.90(0.43$ to 1.89$)$ & 1.69 (0.86 to 3.33$)$ & 0.014 \\
\hline Fatal/non-fatal CHD & 1.0 & $0.98(0.71$ to 1.35$)$ & $1.02(0.74$ to 1.40$)$ & $1.26(0.93$ to 1.71$)$ & 1.56 (1.15 to 2.10$)$ & $<0.001$ \\
\hline Fatal/non-fatal CVD & 1.0 & $0.91(0.71$ to 1.17$)$ & $1.02(0.80$ to 1.31$)$ & 1.18 (0.93 to 1.49$)$ & 1.51 (1.20 to 1.90$)$ & $<0.001$ \\
\hline Cancer deaths & 1.0 & $1.39(0.80$ to 2.43$)$ & $1.20(0.68$ to 2.11$)$ & $1.43(0.83$ to 2.47$)$ & 1.85 (1.10 to 3.13$)$ & 0.027 \\
\hline Lung cancer deaths & 1.0 & 1.66 (0.83 to 3.28$)$ & $1.00(0.47$ to 2.11$)$ & 1.31 (0.64 to 2.66$)$ & $1.76(0.89$ to 3.45$)$ & 0.243 \\
\hline Respiratory deaths & 1.0 & 0.36 (0.09 to 1.36$)$ & $0.65(0.22$ to 1.90$)$ & 0.31 (0.08 to 1.20$)$ & 0.59 (0.20 to 1.78$)$ & 0.310 \\
\hline
\end{tabular}

CRP, C-reactive protein; CHD, coronary heart disease; CVD, cardiovascular disease.

${ }^{*}$ Geometric mean value for each quintile of CRP.

thinear trend from quintile 1 to quintile 5 .

All values are mean $(95 \% \mathrm{CI})$ and have been adjusted for sex, race, age (in quintiles), body mass index (in quintiles), pack-years of smoking (in quintiles), biochemically validated smoking status (continued smokers, sustained quitters, or intermittent quitters), rate of decline in FEV (in quintiles), and predicted FEV (in quintiles).

potential confounders such as age, race, sex, cigarette smoking, and $\mathrm{FEV}_{1}$ (both $\mathrm{FEV}_{1}$ at baseline and the rate of decline in $\left.\mathrm{FEV}_{1}\right)$, indicating that serum CRP provides incremental prognostic information to these traditional markers of morbidity and mortality. Its discriminative power for all-cause mortality was greatest during the first year of measurement and decayed over time. Combined with age, race, and BMI, CRP levels produced a C statistic of 0.82 for l year mortality, suggesting the potential usefulness of serum CRP levels in estimating the prognosis of patients with COPD. In addition, serum CRP levels were significantly associated with cardiovascular events (both fatal and non-fatal) and with cancer specific causes of mortality.

The results of the current study have several implications. Firstly, the findings are consistent with the hypothesis that COPD is a multi-component disease and that systemic inflammation is associated with overall morbidity and mortality in these patients. ${ }^{5}{ }^{6}$ Secondly, high sensitivity CRP assays are now widely available for commercial use so clinicians taking care of COPD patients can use CRP values, in addition to $\mathrm{FEV}_{1}$, to potentially identify patients at high risk of future morbidity and institute early interventional strategy to modify the risk. Thirdly, CRP data may be useful to clinicians to promote smoking cessation as COPD is largely caused by cigarette smoking. ${ }^{1}$ Public awareness of health problems related to cigarette smoking and public health campaigns for smoking cessation have made a significant impact in lowering smoking rates. Nevertheless, 20-25\% of the adult population continue to smoke. ${ }^{16}$ Since active cigarette smoking increases CRP levels, ${ }^{17}$ the findings of this study can be used by health professionals to demonstrate to patients the importance of smoking cessation and in taking up healthy lifestyle choices (such as regular exercise and weight reduction) which together can reduce CRP levels. ${ }^{18} 19$

CRP is a circulating pentraxin that is largely (but not exclusively) produced by hepatocytes as part of an acute phase response. ${ }^{20}$ Although CRP is clearly an excellent and stable biomarker for low grade systemic inflammation, there is no consensus on whether it plays a critical role in mediating chronic inflammatory disorders such as atherosclerosis. ${ }^{20}$ In vitro studies have shown that CRP can activate the classical complement cascade, upregulate adhesion molecules and chemoattractant chemokines, and induce the synthesis of inflammatory cytokines such as interleukin (IL)8 and IL-6 which collectively can amplify the initial inflammatory signal and propagate chronic inflammatory disorders. ${ }^{20}$ Transgenic mouse models of CRP, however, have produced inconsistent results. Notwithstanding the ongoing controversy regarding the potential causal role of CRP, serum CRP levels correlate well with future risk of morbidity and mortality in the general population ${ }^{21} 22$ and in select patient populations such as those with underlying ischaemic heart disease or stroke. ${ }^{23} 24$

There are several limitations to this study. Firstly, the LHS cohort comprised patients who had mild to moderate COPD. It is possible that these results may not apply to individuals without COPD or to those with more advanced disease. Although cardiovascular disease is the leading cause of hospitalisation and one of the leading causes of mortality in mild COPD, respiratory insufficiency and pneumonia become more important in severe COPD. ${ }^{25}$ Whether CRP has a similar discriminative value in patients with more advanced COPD is unknown. Secondly, CRP levels were measured only once in the LHS cohort so we could not evaluate the effect of changes

Table 4 Annual change in $\mathrm{FEV}_{1}$ (\% predicted) in the Lung Health Study cohort according to quintiles of CRP levels and smoking status

\begin{tabular}{|c|c|c|c|c|c|c|}
\hline & Quintile 1 & Quintile 2 & Quintile 3 & Quintile 4 & Quintile 5 & $\begin{array}{l}p \text { for } \\
\text { trendt }\end{array}$ \\
\hline Mean CRP $(\mathrm{mg} / \mathrm{l})^{*}$ & 0.21 & 0.73 & 1.39 & 2.58 & 7.06 & \\
\hline All participants & $0.43(1.69)$ & $0.63(1.50)$ & $-0.58(1.62)$ & $-0.81(1.53)$ & $-0.93(1.54)$ & $<0.001$ \\
\hline Sustained smokers & $1.00(1.60)$ & 1.07 (1.44) & $-1.03(1.57)$ & $-1.30(1.42)$ & $-1.25(1.52)$ & 0.002 \\
\hline Intermittent smokers & $0.10(1.60)$ & $0.30(1.37)$ & $-0.38(1.48)$ & $-0.50(1.52)$ & $-0.64(1.44)$ & $<0.001$ \\
\hline Sustained quitters & $0.43(1.57)$ & $0.15(1.41)$ & $0.37(1.47)$ & $0.12(1.37)$ & $-0.15(1.44)$ & 0.002 \\
\hline
\end{tabular}

*Geometric mean value for each quintile of CRP

thinear trend from quintile 1 to quintile 5 .

The interaction term for smoking status and CRP quintiles was not significant $(p=0.140)$.

Data are expressed as mean (SD) and are calculated from (FEV $1 \%$ predicted in year $1-\mathrm{FEV}_{1} \%$ predicted in year 5$) / 5 y e a r s$ 
in CRP on health outcomes in COPD patients. However, it is assuring that CRP levels measured at multiple time points have been stable in many studies. ${ }^{26-28}$ Moreover, any random fluctuations in CRP levels would have produced nondifferential misclassification, leading to a dilution of the association between CRP and health outcomes. Consistent with this notion, the highest discriminative power of CRP was observed with 1 year mortality and its discriminative power decayed with longer duration of follow up. These data suggest that baseline CRP levels are best used to predict prognosis over a short period of time. Thirdly, we did not measure markers of systemic inflammation other than CRP. We chose CRP because it is a stable molecule with a half life of 18-24 hours, it is raised in COPD, it is easy to measure, and because it has been shown to provide prognostic information in the general population. ${ }^{28} 29$ The importance and usefulness of other markers of systemic inflammation in COPD are much more controversial. Moreover, their assays are not widely available commercially, making them less useful for clinical purposes. Fourthly, it is controversial whether CRP is an effector molecule in the pathogenesis of cardiovascular events or merely a marker of systemic inflammation. The current study was not designed to address this issue. Future work is needed to identify the potential pathogenic role of CRP and other inflammatory mediators in COPD and the conditions associated with COPD. Finally, although we carefully minimised the number of covariates evaluated in the analytical model to mitigate the risk of statistical overfitting, $^{30}$ we cannot fully discount this possibility. Validation of the present findings using a separate COPD cohort is needed in the future.

In summary, the results of the current longitudinal study indicate that the serum level of CRP is a significant predictor of future risk of death, cancer deaths, and cardiovascular events in patients with mild to moderate COPD and provides incremental information beyond that of smoking, $\mathrm{FEV}_{1}$, and other traditional risk factors in COPD. These data suggest that CRP could be used routinely in clinical practice to risk stratify patients with COPD. In those with raised CRP levels, physicians should consider aggressive treatments-for example, smoking cessation programmes, other lifestyle interventions, and possibly some pharmacological agents-to reduce the risk of morbidity and mortality in such patients.

\section{Authors' affiliations}

S F P Man, D D Sin, The James Hogg iCAPTURE Center for Cardiovascular and Pulmonary Research and St Paul's Hospital, Vancouver, British Columbia, Canada

S F P Man, D D Sin, Department of Medicine (Pulmonary Division), University of British Columbia, Vancouver, British Columbia, Canada J E Connett, University of Minnesota School of Public Health, Minneapolis, MN, USA

N R Anthonisen, University of Manitoba, Winnipeg, Manitoba, Canada R A Wise, Johns Hopkins University School of Medicine, Baltimore, Maryland, USA

D P Tashkin, University of California at Los Angeles School of Medicine, Los Angeles, California, USA

This work was funded by the Canadian Institutes of Health Research (Population Health) and by contract N01-HR-46002 from the Division of Lung Diseases of the National Heart, Lung, and Blood Institute (The Lung Health Study).

Competing interests: DDS, SFM, and RW have received research funding from GlaxoSmithKline (GSK) and have received honoraria for speaking engagements from GSK and AstraZeneca.

\section{REFERENCES}

1 Pauwels RA, Buist AS, Calverley PM, et al. Global strategy for the diagnosis, management, and prevention of chronic obstructive pulmonary disease. NHLBI/WHO Global Initiative for Chronic Obstructive Lung Disease (GOLD) Workshop summary. Am J Respir Crit Care Med 2001;163:1256-76.

2 Murray CJ, Lopez AD. Alternative projections of mortality and disability by cause 1990-2020: Global Burden of Disease Study. Lancet 1997;349:1498-504.

3 Andreassen $\mathrm{H}$, Vestbo J. Chronic obstructive pulmonary disease as a systemic disease: an epidemiological perspective. Eur Respir J 2003;46(Suppl):2-4s.

4 Gan WQ, Man SF, Senthilselvan A, et al. Association between chronic obstructive pulmonary disease and systemic inflammation: a systematic review and a meta-analysis. Thorax 2004;59:574-80.

5 Wouters EF. Chronic obstructive pulmonary disease - 5: Systemic effects of COPD. Thorax 2002;57:1067-70.

6 Sin DD, Man SF. Why are patients with chronic obstructive pulmonary disease at increased risk of cardiovascular diseases? The potential role of systemic inflammation in chronic obstructive pulmonary disease. Circulation 2003;107:1514-9.

7 Engstrom G, Wollmer P, Hedblad B, et al. Occurrence and prognostic significance of ventricular arrhythmia is related to pulmonary function: a study from "men born in 1914", Malmo, Sweden. Circulation 2001;103:3086-91.

8 Engstrom G, Lind P, Hedblad B, et al. Lung function and cardiovascular risk: relationship with inflammation-sensitive plasma proteins. Circulation 2002; 106:2555-60.

9 Donaldson GC, Seemungal TA, Patel IS, et al. Airway and systemic inflammation and decline in lung function in patients with COPD. Chest 2005; 128: 1995-2004.

10 Anthonisen NR, Connett JE, Kiley JP, et al. Effects of smoking intervention and the use of an inhaled anticholinergic bronchodilator on the rate of decline of FEV1. The Lung Health Study. JAMA 1994;272:1497-505.

11 Anthonisen NR, Connett JE, Enright PL, et al. Hospitalizations and mortality in the Lung Health Study. Am J Respir Crit Care Med 2002;166:333-9.

12 Crapo RO, Morris AH, Gardner RM. Reference spirometric values using techniques and equipment that meet ATS recommendations. Am Rev Respir Dis 1981;123:659-64.

13 Anthonisen NR, Skeans MA, Wise RA, et al. The effects of a smoking cessation intervention on 14. 5-year mortality: a randomized clinical trial, Ann Intern Med 2005; 142:233-9.

14 Anthonisen NR, Connett JE, Murray RP. Smoking and lung function of Lung Health Study participants after 11 years. Am J Respir Crit Care Med 2002; 166:675-9.

15 Hosmer DW, Lemeshow S. Applied logistic regression, 2nd ed. New York, NY: John Wiley \& Sons, 2000.

16 National Center for Health Statistics. Health, United States, 2004 with chartbook on trends in the health of Americans, Hyattsville, Maryland, 2004.

17 Gan WQ, Man SF, Sin DD. The interactions between cigarette smoking and reduced lung function on systemic inflammation. Chest 2005; 137:558-64.

18 Tchernof A, Nolan A, Sites CK, et al. Weight loss reduces C-reactive protein levels in obese postmenopausal women. Circulation 2002;105:564-9.

19 Esposito K, Pontillo A, Di Palo C, et al. Effect of weight loss and lifestyle changes on vascular inflammatory markers in obese women: a randomized trial. JAMA 2003;289:1799-804.

20 Pepys MB, Hirschfield GM. C-reactive protein: a critical update. J Clin Invest 2003;111:1805-12.

21 Tuomisto K, Jousilahti P, Sundvall J, et al. C-reactive protein, interleukin- 6 and tumor necrosis factor alpha as predictors of incident coronary and cardiovascular events and total mortality. A population-based, prospective study. Thromb Haemost 2006:95:511-8.

22 Tice JA, Browner W, Tracy RP, et al. The relation of C-reactive protein levels to total and cardiovascular mortality in older U.S. women. Am J Med 2003; 114:199-205.

23 Suleiman $M$, Khatib R, Agmon $Y$, et al. Early inflammation and risk of longterm development of heart failure and mortality in survivors of acute myocardial infarction predictive role of C-reactive protein. J Am Coll Cardiol 2006;47:962-8.

24 Kuo HK, Yen CJ, Chang CH, et al. Relation of C-reactive protein to stroke, cognitive disorders, and depression in the general population: systematic review and meta-analysis. Lancet Neurol 2005;4:371-80.

25 Celli BR, Cote CG, Marin JM, et al. The body-mass index, airflow obstruction, dyspnea, and exercise capacity index in chronic obstructive pulmonary disease. N Engl J Med 2004;350:1005-12.

26 Ockene IS, Matthews CE, Rifai N, et al. Variability and classification accuracy of serial high-sensitivity $\mathrm{C}$-reactive protein measurements in healthy adults. Clin Chem 2001;47:444-50.

27 Macy EM, Hayes TE, Tracy RP. Variability in the measurement of C-reactive protein in healthy subjects: implications for reference intervals and epidemiological applications. Clin Chem 1997;43:52-8.

28 Ridker PM, Cannon CP, Morrow D, et al. C-reactive protein levels and outcomes after statin therapy. N Engl J Med 2005;352:20-8.

29 Libby P, Ridker PM. Inflammation and atherosclerosis: role of C-reactive protein in risk assessment. Am J Med 2004;116(Suppl 1):9-16S.

30 Selker HP. Systems for comparing actual and predicted mortality rates: characteristics to promote cooperation in improving hospital care. Ann Intern Med 1993;118:821-3. 